\title{
Téoros
}

Revue de recherche en tourisme

\section{Regards neufs sur les attractions touristiques du Québec}

\section{Marc Laplante}

Volume 2, numéro 2, juin 1983

Regards neufs sur les attractions touristiques du Québec

URI : https://id.erudit.org/iderudit/1080830ar

DOI : https://doi.org/10.7202/1080830ar

Aller au sommaire du numéro

Éditeur(s)

Université du Québec à Montréal

ISSN

0712-8657 (imprimé)

1923-2705 (numérique)

Découvrir la revue

Citer ce document

Laplante, M. (1983). Regards neufs sur les attractions touristiques du Québec. Téoros, 2(2), 1-1. https://doi.org/10.7202/1080830ar d'utilisation que vous pouvez consulter en ligne.

https://apropos.erudit.org/fr/usagers/politique-dutilisation/ 


\section{Regards neufs}

\section{sur les attractions touristiques du Québec}

Quand le gouvernement québécois, au nom de toute la population, proclame a pleins poumons que le Quebec. "C'est beau, c'est grand, c'est à voiry, qui prendra le risque d'affirmer pubhquement que ce méme pays n'est pas une destination touristique très attirante? En privé cependant, et aussi a l'occasion de certains congrès ou colloques, nous entendons parfois des lamentations du genre: le Quebec est winvendablen (touristiquement), ce que te Québec a de plus original (les grands espaces, I'hiver, le St-Laurent, la culture francophone, etc. ) est peu rprésentabley aux touristes, certains des attraits du Quebec qui ont fait la fortune du tourisme dans le passé se sont "dégradésy (par exemple, les clubs privés de chasse et de péche, la vie rurale et villageoise), etc.

De telles idées expriment un malaise. Le Quebec, privé de sa clientele étasunienne depuis la première crise du pétrole, cherche à tâton de nouvelles voies pour intéresser et séduire de nouveaux publics touristiques. Le cheminement par (ressai et erreury est long et décourageant. Que des doutes naissent alors sur la valeur attractive du produit touristique lui-même et précisément, des attraits et attractions qui peuvent influencer le choix d'une destination de vacances, rien de très étonnant. Qu'on tente aussi de miser davantage sur le tourisme de congrès n'est guére surprenant. Qu'on s'interroge enfin - comme nous tentons de le faire avec ce présent numero de Téoros - sur les qualités du produit touristique québécois apparaîtra comme une tâche pertinente à ce moment-ci du développement touristique chez nous.

Qu'est-ce qui attire les touristes au Québec? Ou plutôt, qu'est-ce qui les attirait jadis et qu'est-ce qui pourrait les attirer dans /avenir? Suffira-t-il, comme plusieurs intervenants le prétendent aujourd'hui, de redevenir plus concurrentiels au niveau des couts des services (hotellerie, restauration, transport/? Faut-l/ investir davantage encore dans les infrastructures (Palais des congrès, super-stations du Mont Tremblant, du Mont Ste-Anne ou du Mont Orford, etc. I comme nous le faisons présentement? Est-il préférable - pour un temps du moins - de restreindre les sorties des Québecois hors de nos frontieres pour les inciter davantage à prendre des vacances au Quebec?
Ces mesures - et d'autres semblables - pourront peutetre ameliorer la situation a court terme. Sur une plus longue période cependant, nous crovons nécessaire une remise en question courageuse de nos habitudes de penser face aux touristes et au tourisme. /l nous faudra surtout comprendre le touriste, savoir ce qu'il cherche et ce qu'il attend en voyage de vacances. Plus cette compréhension s'accroitra, plus les transformations du produit touristique seront pertinentes et rentables.

$A$ titre de contribution à ce renouveau des idees sur le tourisme, nous proposons dans les pages qui suivent quelques textes variés sur les attractions touristiques du Québec. En apparence, une étude des attractions releve d'une analyse de /'offre touristique. Nous aurons /'occasion de constater cependant que le modele couramment emplové de l'offre et de la demande masque des dimensions trés intéressantes de la réalité touristique. Nous proposons p/utót l'approche des sciences de la communication qui oblige l'ceil a s'attarder sur les interactions entre le producteur-émetteur et le consommateur-récepteur. Ainsi, nous centrerons nos réflexions sur le couple: attractiontouriste et sur le contexte de ces interactions.

Nous invitons donc le public lecteur de Téoros, quand il partira en vacances a son tour, a jeter un regard neuf sur les touristes et les attractions quills croiseront en route. Les pages théoriques et pratiques de ce numéro posent de nombreuses questions. Nos réponses ne peuvent etre que partielles; les observations libres et attentives de la réalité par tous ceux et celles que le tourisme passionne viendront les completer.

Marc Laplante. 